\title{
CJEM Debate Series: \#SocialMedia - Social media has created emergency medicine celebrities who now influence practice more than published evidence
}

Peter Cameron, MBBS, MD*; Simon Carley, MB ChB, MPhil, MD ; Scott Weingart, MD*; Paul Atkinson, MB BCh BAO, MA ${ }^{\S}$

\section{INTRODUCTION}

\section{Paul Atkinson (@eccucourse @sjrhem)}

This series of editorials will provide CFEM readers with the opportunity to hear differing perspectives on topics pertinent to the practice of emergency medicine. The debaters have been allocated opposing arguments on topics where there is some controversy or perhaps scientific equipoise.

We continue with the topic of social media and celebrity, a topic key to how we progress information sharing in academic and clinical emergency medicine. Have new online platforms made traditional publication redundant? Have we abandoned evidence-based medicine and peer review and created social media celebrities who now influence our practice? Or is this phenomenon a natural progression that complements traditional forms of information sharing, showing the progressive nature of our specialty?

Dr. Peter Cameron, former President of the International Federation for Emergency Medicine, academic, and clinician, makes the argument that social media presents us with the dangerous potential of style over content as he argues for the motion, with Drs. Simon Carley and Scott Weingart, both active academics, clinicians, and social media leaders, responding to reassure us that newer forms of online information sharing will help us improve what we do, and respect an evidence-based approach.

Readers can follow the debate on Twitter and vote for either perspective, by going to @CFEMonline or by searching \#CFEMdebate.
SOCIAL MEDIA HAS CREATED EMERGENCY MEDICINE CELEBRITIES WHO NOW INFLUENCE PRACTICE MORE THAN PUBLISHED EVIDENCE

\section{For: Peter Cameron (@prof_cameron)}

The power and potential risks of the combination of social media and celebrity status are evident in recent international events. Politicians ruthlessly manipulate public opinion and celebrity status in gaining power and influencing public debate. The use of Twitter, Instagram, and Facebook to circumvent conventional media and "speak directly to the people" is now routine. In the recent U.S. election, a much better funded and heavyweight political organization was left flat-footed in the face of this phenomenon. Similar observations could be made regarding the development of terrorist groups and other unconventional political organizations. Governments of the major powers are accusing each other of manipulating messages to covertly influence public policy in their countries. As scientists and clinicians with a passion for practicing the best emergency medicine according to the best evidence, are we immune from these powerful forces? Social media has been promoted as a form of democratization in the market place of ideas and innovation. ${ }^{1}$ Is this true? Many would argue that social media simply represents a cynical process for controlling the message through constant bombardment, repetition, selective reporting, and overt misrepresentation of events and observations ${ }^{2}$ - the recipient being unaware of his or her naïve acceptance of what is clearly a controlled version of "the truth."

From the *Emergency and Trauma Centre, The Alfred Hospital, Melbourne, Victoria, Australia; †Central Manchester NHS Foundation Trust, Manchester, UK; ¥Stony Brook Medicine, Stony Brook, NY; and §Dalhousie University, Saint John Regional Hospital, Saint John, NB.

Correspondence to: Dr. Paul Atkinson, Emergency Medicine, Saint John Regional Hospital, 400 University Ave., Saint John, NB E2L 4L4; Email: paul.atkinson@dal.ca 


\section{What is social media?}

From Wikipedia: ${ }^{3}$

The variety of stand-alone and built-in social media services currently available introduces the challenges of defining. However, there are some common features.

1) Social media are interactive Web 2.0 Internet-based applications.

2) User-generated content, such as text posts or comments, digital photos or videos, and data generated through all online interactions, are the lifeblood of social media.

3) Users create service-specific profiles for the website or app that are designed and maintained by the social media organization.

4) Social media facilitate the development of online social networks by connecting a user's profile with those of other individuals and/or groups.

This diverges from traditional print or online published material, because there is immediacy and accessibility that enables recipients to be current with all innovations and developments at all times. This immediacy and accessibility is traded against the negative attributes of the inability to crosscheck with previous literature, verify source data, check credentials of the authors, and review methodology. A limit of 140 characters (or similar brevity) enables rapid updates, but no details. There is an immediacy, measured in seconds, that has not previously been possible.

The converse of immediacy is delayed and considered application of new findings and innovations. Is it important that all new information is implemented immediately? Does it matter that a consensus review might reverse conclusions 1 or 2 years later? Evidence-based medicine advocates bemoan the fact that new drugs and interventions take an average of 17 years to become mainstream. ${ }^{4}$ Social media offer the opportunity to shorten this by years through increased connectivity. Is this not a good thing?

The reality is that we are currently using social media in an uncontrolled and naïve way. Residents (and consultants) read the latest blog from an "expert celebrity" without checking what the full facts are behind this opinion. It is similar to the "click bait" news that we are all subject to on our mobile phones. Why are some pieces of information broadcast and others not? The propagator of the "news" always has an agenda - fame, fortune, promotion. Sometimes this motivation is evident, sometimes not. For speakers and journal authors, we have strict rules for disclosure; there is little consequence for bloggers and tweeters (you may even become POTUS).

What is re-posted and what "goes viral" very much depends on glitz rather than importance to patient outcome. Unfortunately, major organizations such as the American, Canadian, and Australasian emergency medicine colleges or associations (ACEP, CAEP and ACEM) may inadvertently be used as vehicles for promoting unbalanced reporting of new evidence. It is very hard to have 24/7 "fact-checking" and review of posted material. So what is "trending" (as in the most popular hits) can be easily manipulated and accelerated by obsessed campaigners, especially those with big budgets. The founder of the Internet, Tim BernersLee, in an open letter, expressed his concern regarding "fake news" - "The net result is that these sites show us content they think we'll click on, meaning that misinformation, or fake news which is surprising, shocking or designed to appeal to our biases can spread like wildfire. And through the use of data science and armies of bots, those with bad intentions can game the system to spread misinformation for financial and political gain..."

We have entered a new era with new terminology, "post truth" and "fake news." It seems that reality and evidence are less important than fame and celebrity. As clinicians and scientists, we should be smarter than the general public in responding to $24 / 7$ tweets, blogs, and Facebook messages. We should have access to sites that are reputable, based on scientific consensus, and verified by a collective of our most informed, scientifically rigorous colleagues. The Internet has given us unparalleled access to reference tables and texts as well as consensus statements from internationally respected organizations. It is incumbent on the responsible, independent clinical organizations to promote their online references. Unrestricted use of social media by uninformed consumers creates celebrities, fake news, and impedes considered deliberation of innovation in health care. A tool to "democratize" news is now making it more difficult to understand the importance of "breakthroughs." The historical, derogatory term, eminence-based medicine, has been replaced by celebrity medicine, and evidence is now a relative term. 
SOCIAL MEDIA HAS CREATED EM CELEBRITIES WHO NOW INFLUENCE PRACTICE MORE THAN PUBLISHED EVIDENCE

\section{Against: Simon Carley (@EMManchester @stemlyns) and Scott Weingart (@emcrit)}

\section{Anyone who tries to make a distinction between edu- cation and entertainment doesn't know the first thing about either.}

\section{- Marshall McLuban}

It takes an estimated 12 to 17 years for high-quality evidence to reach our patients. ${ }^{6}$ In other words, the traditional model of journal publication in its vain hope of disseminating wisdom far and wide to the benefit of our patients is broken. It is therefore no surprise that educators and researchers have embraced new technological tools to speed this knowledge translation gap in the hope that we might do better.

Despite the obvious and well-documented flaws in the traditional publication model, there remain some who seek to defend the traditional, comfortable approach, perhaps because they feel that they have a degree of control over it. They dislike the agility, speed, and broad reach of social media enabled education, but we argue that those are exactly the characteristics that evidence-based medicine needs in a world where our patients deserve clinicians who are up to date and well informed of the latest research.

We should pause and think about what we mean by "published evidence." Simply publishing an article in a journal implies quality, but that is far from the truth. Much of what is published is wrong, misleading, or is simply worthless to real world clinical practice. The prepublication process of peer review is outdated, prone to fraud, and unable to detect serious errors in methodology or interpretation. ${ }^{8}$ What does this mean for clinicians? Simply put, the volume of literature published means that it is impossible for any individual to filter and appraise all that is relevant to his or her practice, an issue especially pertinent in a specialty as broad as emergency medicine.

Post-publication review, widely shared, in a public space, and with the ability to react in real time is surely a way forward to increase the distribution and interpretation of published evidence, and this is what the current crop of blogs, podcasts, and other social media platforms aim to do under the \#FOAMed (Free Open Access Medical Education) banner, ${ }^{9}$ a more bespoke and focused subset of social media with an aim to improve clinical practice. ${ }^{10}$
The \#FOAMed platforms permit, and arguably encourage, creators and consumers to use data in new ways, to operationalize research and translate it into something that is pragmatic and deliverable in the clinical setting. Clinical practice rarely dictates that any individual decision should be based solely on the result of a paper. Real-world clinical decisions are far more complex than that and require the discussion and interpretation of any published evidence in relation to the clinical setting, cost, patient group, and the abilities of the clinicians who might be required to deliver it. Whilst journals and publishers could deliver such debate and interpretation in print, or even online, the fact is that they don't.

Interpretation is difficult and time-consuming, requiring subject matter expertise in areas of clinical practice that may be beyond those of the original authors. This is where \#FOAMed exists at the interface between clinical practice and published evidence - a role and that has forever been inhabited by those who wish to improve patient outcomes.

This brings us to the issue of what is meant by the term celebrity. By definition, this simply means "being well known." Unsurprisingly, it is then self-evident that those who are well known will have a broader reach to influence others. This in itself is nothing to be scared about, although it might provoke jealously. However, in this context, it apparently has pejorative connotations. If not jealousy, then perhaps it is the content that is being criticized? If so, then the evidence for this is lacking. The \#FOAMed advocates are well known for promoting evidence-based medicine and thinking, with their arguments based in the interpretation of the published literature.

We suspect that celebrity refers to those who are able and willing to talk about clinical practice in a broad, open, and free environment, typically integrating their communication through novel methods (social media) and also through more traditional methods such as conference presentations and journal publication. Those who are well known in the \#FOAMed world are also typically well published in the traditional academic literature; thus, the artificial distinction between the \#FOAMed world and traditional publication is an illusion.

To be well known and able to communicate effectively is surely a good thing in medicine and an essential requirement for evidence-based medicine.

Perhaps, the critics are envious that their unwillingness (or inability) to communicate using modern means is leaving them behind. Are they concerned that their personal contributions are overlooked, as they remain 
trapped in the knowledge translation gap of traditional publication methods? Or is it possible that they are concerned that they are not being recognized as the expert clinicians that they perceive themselves to be? Perhaps it would be rude of us to suggest such narcissistic elitism amongst the critics, but we cannot ignore the possible influence of jealousy or envy as the driver of resistance.

Perhaps the celebrity insult relates to the ability of social media to entertain as well as to educate. This linkage is clearly a difficulty for many who prefer their evidence in dry, boring, formulaic and largely unread passive tense, house style of many medical journals. Do we really believe that this is the best way to communicate in the modern age? Education theory clearly shows that learners achieve more when they are engaged with the medium through which the information flows. In the social age, that means engaging through and within social and electronic spaces. ${ }^{11}$ The modern day human is digitally connected in a way that permeates every aspect of their lives, and we are increasingly accustomed to consuming information from all areas using our phones and computers. To imagine that medical education is immune from this trend is naïve, and we must adapt to it. Marshal McLuhan famously said that as we change the medium through which we access information, then we potentially change the message.

\section{The medium is the message.}

\section{- Marshall McLuban}

In 2017, McLuhan's words seem very prophetic to the digitally enabled, connected, and social age. Modern educators have recognized this and thus changed the way that information is presented to engage and indeed entertain the consumer, in recognition of the intertwining of entertainment and education. For example, could you imagine a podcast based on simply reading out a paper from a journal? That would be ridiculous, but those who criticize social media might argue that we should do this or not use it at all. The medium of learning has clearly changed in health care, but it would seem that not all clinicians have kept apace of this.

Lastly, critics frequently fall into the cognitive trap of suggesting that the consumer is both unable to discern the quality of information from celebrities via social media whilst simultaneously suggesting that if the information was presented in a different (that is, traditional) method they would be perfectly capable of doing so. This is a logical fallacy. How can the consumer only be misled if receiving information in an engaging and electronic medium, or do critics simply think their audience is incapable of independent thought?

Painting the consumer as a naive, unquestioning, and passive participant in the receipt of information is wrong. Suggesting that learners are easily led down the wrong path attracted on the basis of glitz, glamour and sound bites, changing practice without due diligence and care is unfair. We find the elitist arrogance of such attitudes immeasurably insulting to our colleagues who are perfectly capable of exhibiting the judgment and discrimination to decide what they will and will not believe. To argue otherwise would be deeply patronizing to us all.

Competing interests: None declared.

Keywords: social media, evidence based medicine, influence

\section{REFERENCES}

1. Johnson SMB. Unstoppable march of social media and democratization. The Guardian, 15 September 2015. Available at: https:/guardian.ng/features/unstoppable-march-ofsocial-media-and-democratisation/ (accessed 22 March 2017).

2. Hamilton JM, Kosar K. How the American government is trying to control what you think. The Washington Post, 24 September 2015. Available at: https://www.washingtonpost. com/posteverything/wp/2015/09/24/the-new-propaganda-howthe-american-government-is-trying-to-control-what-you-think/ ?utm_term=.52faab7cf282 (accessed 22 March 2017).

3. Social media. Wikipedia. Available at: https://en.wikipedia. org/wiki/Social_media (accessed 22 March 2017).

4. Morris ZS, Wooding S, Grant J. The answer is 17 years, what is the question: understanding time lags in translational research. $7 R$ Soc Med 2011;104(12):510-20.

5. Lee TB. Three challenges for the web, according to its inventor. Web Foundation, 12 March 2017. Available at: http:// webfoundation.org/2017/03/web-turns-28-letter/ (accessed 22 March 2017).

6. Goss JR, Elmore JG, Lessler DS. Quality of health care delivered to adults in the United States. $N$ Engl $7 \mathrm{Med}$ 2003;349(19):1866-8.

7. Chan T, Trueger NS, Roland D, et al. Evidence-based medicine in the era of social media: scholarly engagement through participation and online interaction. CFEM 2017;12:1-6.

8. Smith R. Peer review: a flawed process at the heart of science and journals. $7 R$ Soc Med 2006;99(4):178-82.

9. Nickson CP, Cadogan MD. Free Open Access Medical education (FOAM) for the emergency physician. Emerg Med Australas 2014;26(1):76-83.

10. Thoma B, Mohindra R, Artz JD, et al. CFEM and the changing landscape of medical education and knowledge translation. CFEM 2015;17(2):184-7.

11. Stodd J. A guide to the social age 2016; 2016. Available at: https://julianstodd.wordpress.com/2016/01/06/a-guideto-the-social-age-2016. 